\title{
Fusarium Populations on Chinese Barley Show a Dramatic Gradient in Mycotoxin Profiles
}

\author{
L. Yang, T. van der Lee, X. Yang, D. Yu, and C. Waalwijk
}

First, third, and fourth authors: Institute for Plant Protection and Soil Sciences, Hubei Academy of Agricultural Sciences, 430064, Wuhan, China; and second and fifth authors: Plant Research International BV, P.O. Box 166700 AA, The Netherlands. Accepted for publication 3 February 2008.

\begin{abstract}
Yang, L., van der Lee, T., Yang, X., Yu, D., and Waalwijk, C. 2008. Fusarium populations on Chinese barley show a dramatic gradient in mycotoxin profiles. Phytopathology 98:719-727.

We report on a large gene bank of Fusarium isolates established by a broad survey conducted in 2005 in which infected barley ears were collected in 23 counties of seven provinces and two municipalities along the Yangtze River in China. In total, 1,894 single spore isolates were obtained. The isolates were characterized at the species level by a newly developed and robust set of diagnostic primers based on single nucleotide polymorphisms (SNPs) among members of the $F$. graminearum clade. In addition, we determined their chemotype using previously described polymerase chain reaction (PCR) primers. The results showed that in all

blight (FHB) on barley in China $(\mathrm{N}=1,706)$, while in the upper valleys of the Yangtze River also $F$. graminearum sensu stricto, $F$. meridionale, and $F$. proliferatum were found. Major differences in the chemotypes were found in the $F$. asiaticum populations, from very high to exclusive nivalenol (NIV) chemotypes in the mountainous upper valleys of the Yangtze River to predominantly deoxynivalenol (DON) chemotypes in the middle and lower valleys. In contrast to the $F$. asiaticum isolates from three counties in Sichuan province, which were largely NIV producers (278 of 291), F. graminearum isolates from these sampling sites were for the vast majority (27 of 28) DON producers, indicating that despite sharing the same habitat, these sympatric species apparently have unique mycotoxin chemotypes.
\end{abstract} regions $F$. asiaticum was the predominant species causing Fusarium head
Additional keywords: feed safety, food safety, scab, SNP detection, toxin.
Fusarium head blight (FHB), or scab, is an economically devastating disease of wheat, barley, and other small grain cereals worldwide $(7,19,24,33)$. In China, the disease frequently occurs in wheat and barley growing areas in the middle and lower valleys of the Yangtze River, the mountainous areas in southwest China, and the Heilongjiang plain in northeast China. In these regions humidity is high and temperatures are moderate during heading and flowering of both wheat and barley. These climatic conditions are highly conducive for FHB and consequently this disease is considered a major constrain for wheat production in China. In the last 10 years, FHB resulted in a sharp decrease of wheat growing acreage in the southern part of China. In Hubei province, the wheat growing acreage decreased from 1.2 million ha to less than 0.6 million ha due to the yield and quality reduction caused by FHB in the last decade. The impact was even more dramatic in the Shanghai, Fujian, and Zhejiang provinces where the majority of farmers stopped growing wheat due to FHB epidemics. In the north and northwest provinces of China, such as Shaanxi, Henan, and Shandong, conducive weather conditions occasionally drive outbreaks of FHB, resulting in extensive yield losses in wheat and barley.

FHB is caused by a complex of Fusarium spp. that apart from causing direct and indirect losses, also produce a range of mycotoxins $(2,26)$ which are harmful to humans and livestock $(7)$. Therefore, mycotoxin contamination of wheat, barley, and corn caused by Fusarium spp. is a major public concern globally, and awareness of related food and feed safety issues is growing in China. A variety of mycotoxins are produced by different species

Corresponding author: C. Waalwijk; E-mail address: cees.waalwijk@wur.nl

doi:10.1094/PHYTO-98-6-0719

(C) 2008 The American Phytopathological Society of Fusarium. Members of the F. graminearum clade and F. culmorum produce type B trichothecenes including nivalenol (NIV), deoxynivalenol (DON), and its acetyl derivatives. Because of toxicological differences between these mycotoxins (reviewed by Schlatter [27]), it is important to survey the presence of the different Fusarium species and their chemotypes in a given region. The species composition of Fusarium populations may strongly vary over spatial (23) and temporal scales (31). Up to 17 species have been associated with FHB of wheat in Europe but $F$. graminearum, F. culmorum, F. avenaceum, F. poae, and Microdochium nivale are reported to be the most common pathogens on wheat (24), and similar results were reported in Canada (28). Based on morphological studies, up to 27 different fungal species/ varieties were associated with $\mathrm{FHB}$ on wheat in China but $\mathrm{Fu}$ sarium spp. of the $F$. graminearum clade dominate the populations $(6,17)$. However, no isolates were stored and little is known about the FHB complex on barley in China. In addition, recent research based on random amplified polymorphism DNA (RAPD) and sequence characterized amplified region (SCAR) analyses demonstrated that the monomorphic $F$. graminearum clade is genetically diverse $(3,4)$. More detailed phylogenetic analysis of DNA sequences of 11 nuclear genes, including the mating type locus, revealed that $F$. graminearum consists of at least nine biogeographically structured lineages ( $F$. graminearum clade), which have been formally named $(21,22)$. This refined species definition allows more detailed analyses of Fusarium populations within cereal growing regions. With the identification of the genes responsible for toxin production, PCR has become increasingly popular to discriminate between different chemotypes $(5,13$, $14,30)$. More recently, primer pairs based on the intergenic region between the tri5 and tri6 genes were designed to differentiate between DON and NIV producers (15).

The objectives of this research were to study the cause of FHB on barley in China by comparing populations of Fusarium spp. in 
different barley growing regions along the Yangtze River. Specifically, we (i) conducted a survey on the severity of FHB in barley in different regions in China, (ii) established a large hierarchically sampled collection of Fusarium isolates from barley, (iii) characterized this population at the species level, and (iv) determined the distribution of the different trichothecene chemotypes.

\section{MATERIALS AND METHODS}

Collection of samples and isolation of Fusarium species. Diseased barley spikes with mature kernels were hierarchically sampled 1 to 2 weeks before harvest from 23 counties in seven provinces and two municipalities (Fig. 1). Ten spikes with FHB symptoms were collected individually at five different positions in each field. Kernels of collected barley spikes were surface sterilized in $70 \%$ ethanol for $30 \mathrm{~s}$, and immediately immersed in $2 \%$ sodium hypochlorite for $90 \mathrm{~s}$, after which the kernels were extensively rinsed with sterile distilled water and placed on potato dextrose agar (PDA) plates. After 3 days of incubation at $26^{\circ} \mathrm{C}$, newly grown-out mycelium was transferred into flasks containing $30 \mathrm{ml}$ of autoclaved mung bean broth. This medium was prepared essentially according to Bai and Shaner (1) with the modification that the beans were removed from the boiling mixture immediately after the very first peels became detached. Flasks were shaken at $110 \mathrm{rpm}$ for 4 to 5 days at $26^{\circ} \mathrm{C}$. Spore suspensions were diluted to 1,000 -fold and plated on $1 \%$ water agar. The plates were incubated for 12 to $24 \mathrm{~h}$ and single spores were identified and transferred to PDA plates. Single spore cultures were stored in $15 \%$ dimethyl sulfoxide (DMSO) at $-80^{\circ} \mathrm{C}$.

DNA extraction. A small mycelial plug was transferred to petri plates (diameter of $6 \mathrm{~cm}$ ) containing potato dextrose broth (PDB) and incubated at $25^{\circ} \mathrm{C}$ for 5 to 7 days. Mycelium was harvested with a toothpick, transferred to 96 well format blocks and then stored at $-80^{\circ} \mathrm{C}$ until DNA extraction. After addition of a tungsten bead, the mycelium was lyophilized and ground by vigorous shaking of the blocks in an MM300 mixer mill (Retch, Ochten, The Netherlands). Total genomic DNA was extracted using the Genomic DNA Extraction Kit (Puregene DNA Purification, Gentra) according to the manufacturer's instructions. After electrophoresis on $1.0 \%$ agarose gels, the DNA concentration was estimated by comparison with a serial dilution of known amounts of DNA. Finally, DNA samples were diluted to $2 \mathrm{ng} / \mu \mathrm{l}$ for each sample in 96 -well microtiter plates which were stored at $-20^{\circ} \mathrm{C}$.

Design of species-specific polymerase chain reaction (PCR) primers. To resolve the different members of the $F$. graminearum clade (for brevity from hereon called the $F$. graminearum clade) we designed a competition PCR strategy. This PCR is designed to enhance the specificity of the PCR amplification and to improve scoring. We used the species-specific primers in combination with generic primers, whereby the species-specific primers compete with the generic primers. Without a perfect match for the speciesspecific primers, only the generic primers will generate an amplicon with a size different from the amplicon generated by the species-specific primers. In this way single nucleotide polymor-

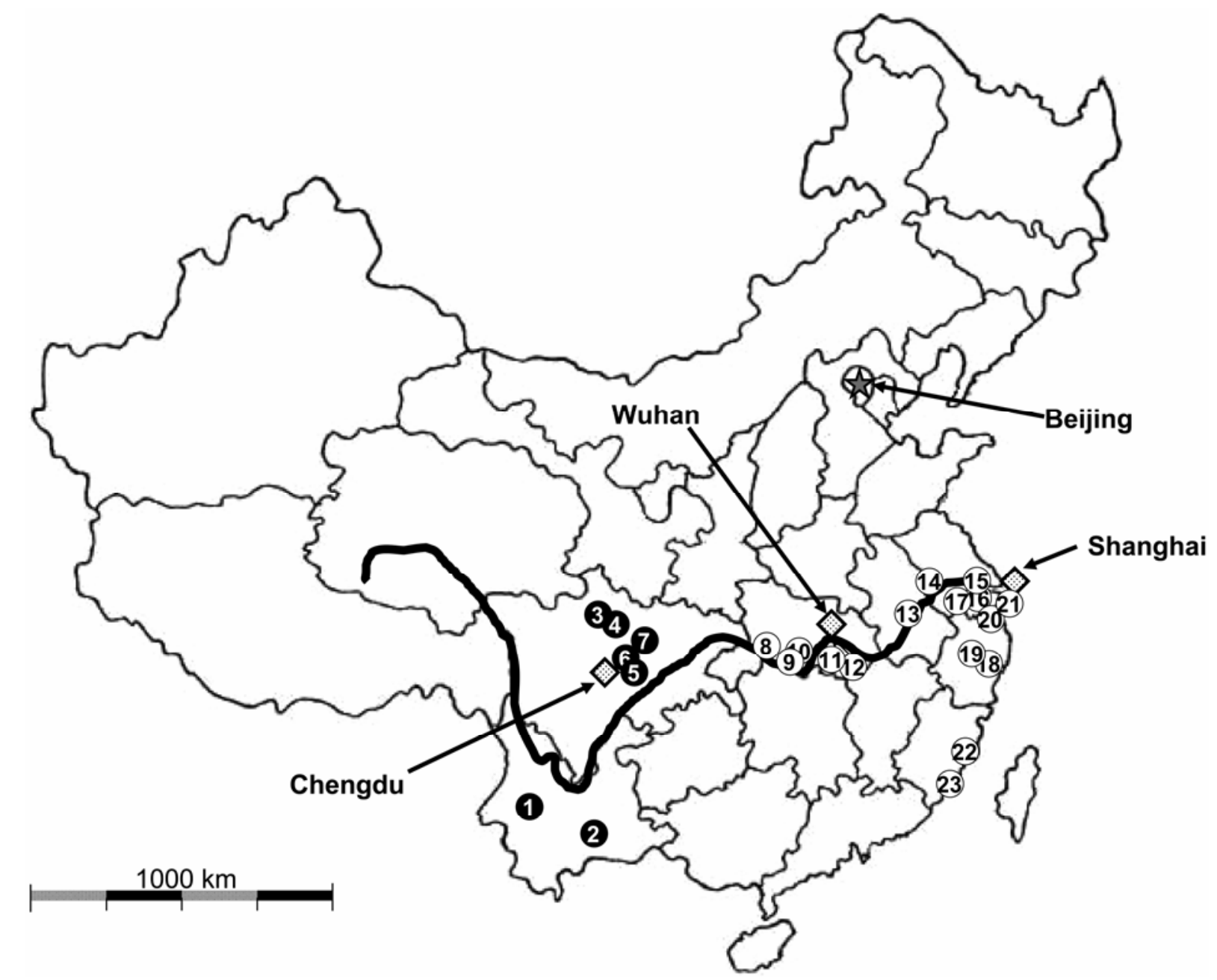

Fig. 1. Map of China indicating the sampling sites along the Yangtze River (bold line) used for the collection of Fusarium isolates of the Barley Fusarium Bank. Numbers indicate the counties and correspond with the numbering in Table 2. Sampling sites at higher altitudes $(>250 \mathrm{~m})$ are indicated with dark circles and those at sea level with open circles. Major cities are also indicated. 
phisms (SNPs) between members in the $F$. graminearum clade are translated into PCR amplicons of different lengths (Fig. 2).

Partial sequences of the ammonia ligase 2 (CTPS2) gene $(557 \mathrm{bp})$ and the translation elongation factor $1 \alpha$ (TEF-1 $\alpha)$ gene (644 bp) of 45 Fusarium isolates, representing F. culmorum, F. cerealis, $F$. pseudograminearum, $F$. lunulosporum, and nine species of the $F$. graminearum clade (e.g., F. acaciae-mearnsii, $F$. asiaticum, $F$. austroamericanum, $F$. boothii, $F$. brasilicum, $F$. cortaderiae, $F$. graminearum sensu stricto, $F$. meridionale, and $F$. mesoamericanum) were collected from the NCBI database. Se- quences were aligned with the Megalign module of DNAstar 6.0 and species-specific SNPs (22) were recognized. In the partial sequence of the ammonia ligase 2 gene, there is an adenosine at $\mathrm{nt}$ 177 in representative isolates of F. asiaticum (e.g., NRRL6101) while in all other species tested there is a cytosine at this position (Fig. 2). Based on this SNP, we designed a primer specific for $F$. asiaticum. In addition, $F$. meridionale isolates have a thymidine at nt 306 in the partial sequence of ammonia ligase 2 gene, while there is a cytosine in the other species of the $F$. graminearum clade. Based on this SNP, we designed a primer specific for $F$.
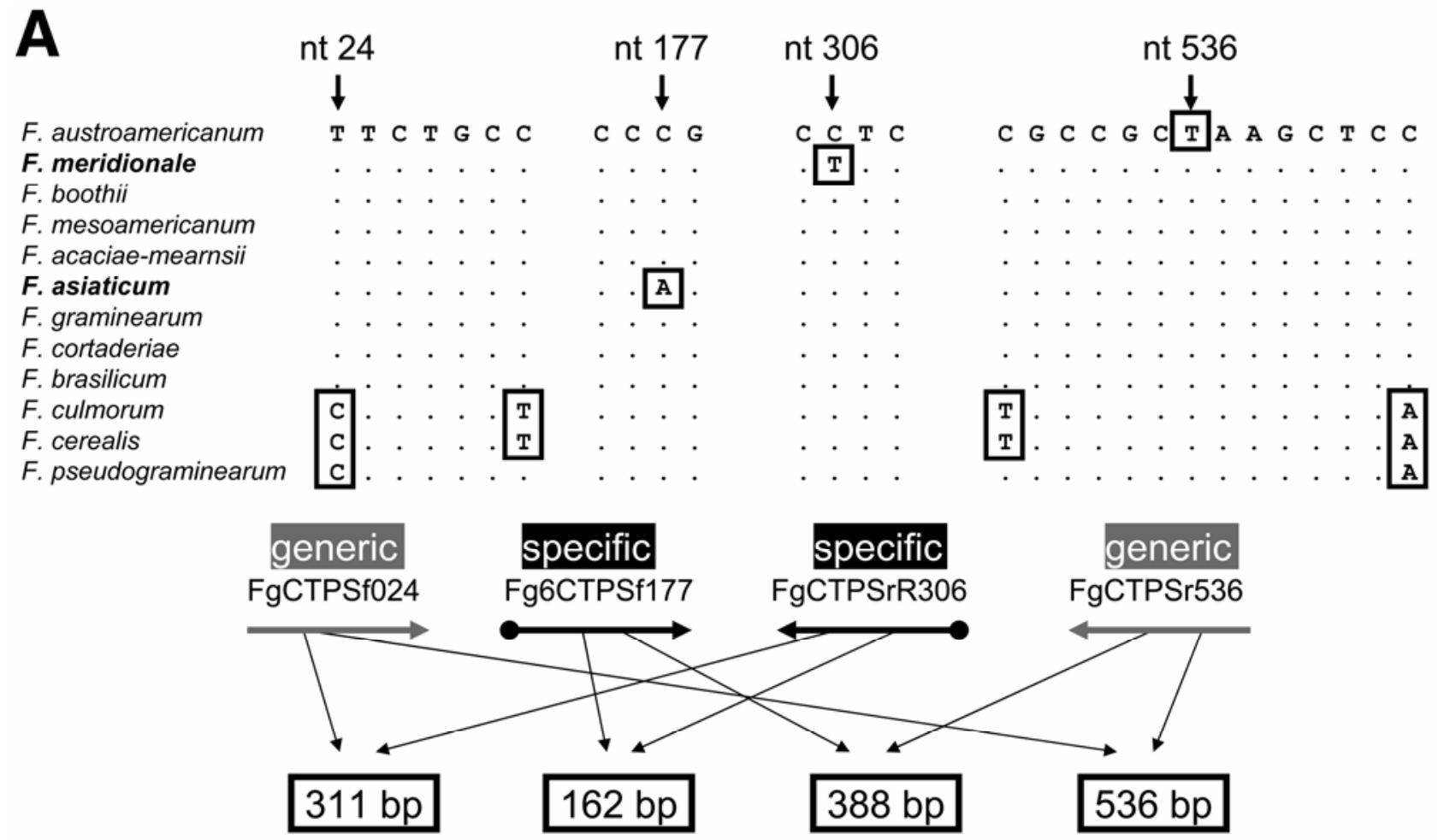

B
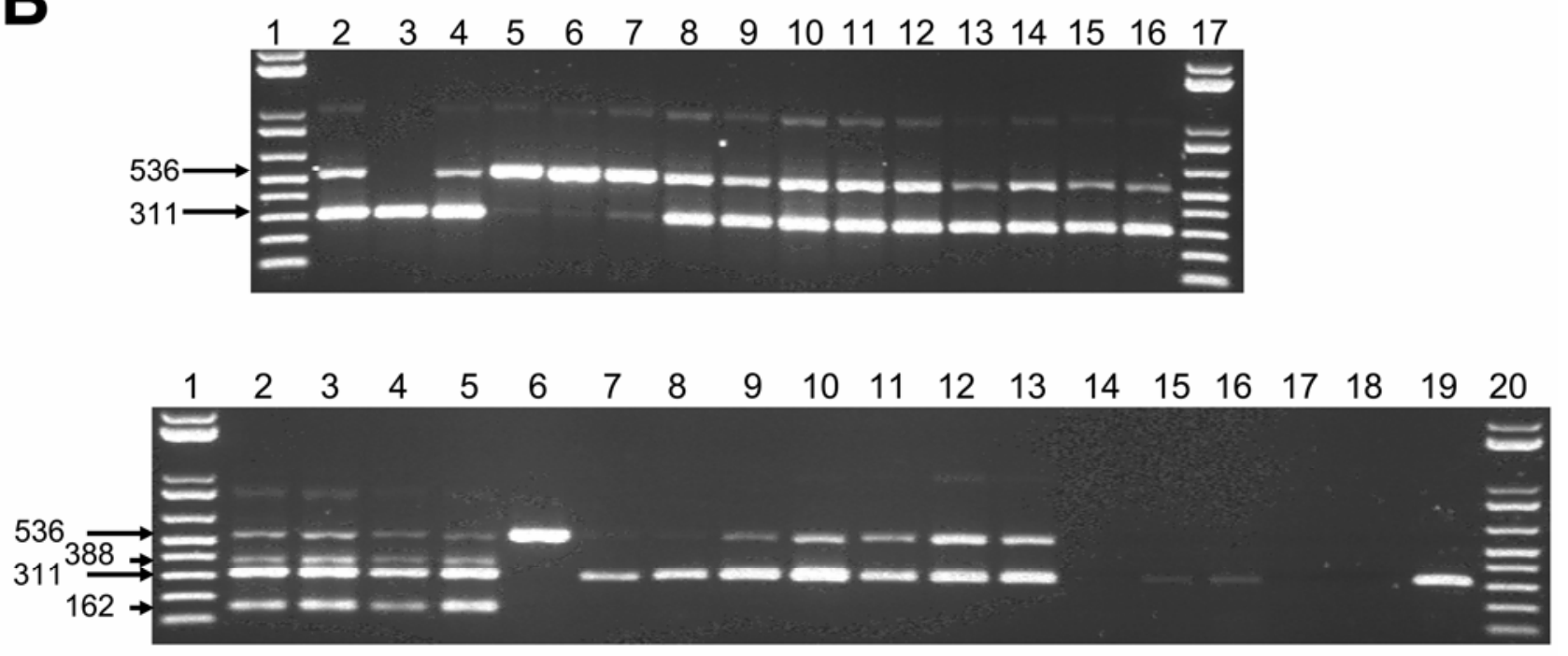

Fig. 2. A, An example of a competition polymerase chain reaction with primers FgCTPSf024, FgCTPSr536, Fg6CTPSf177, and FgCTPSr306 performed on a reference set of Fusarium species. B, Top gel: lanes 1 and 17, molecular size markers; lanes 2 to 4, F. austroamericanum NRRL 28585, NRRL 28718, and NRRL28436; lanes 5 to 7, F. meridionale NRRL 28723, NRRL 29010, and NRRL 29020; lanes 8 to 11, F. boothii NRRL 26916, NRRL29011, NRRL 29105, and NRRL 25797; lanes 12 and 13, F. mesoamericanum NRRL 29148 and NRRL 26752; lanes 14 to 16 F. acaciae-mearnsii NRRL 26752, NRRL 26754, and NRRL 26755. Bottom gel: lanes 1 and 20, molecular size markers; lanes 2 to 6, F. asiaticum NRRL 6101, 13818, 26156, 28720, and 28721; lanes 7 to 13, F. graminearum NRRL 5883, NRRL 6394, NRRL 13383, NRRL 28063, NRRL 28336, NRRL 28439, and NRRL 29169; lanes 14 to 15, F. culmorum NRRL3288 and NRRL 25475; lanes 16 to 18, F. cerealis NRRL 13721, NRRL 25491, and NRRL 25805; and lane 19, F. lunulosporum NRRL 13393. 
meridionale and a primer for all members of the $F$. graminearum clade except for $F$. meridionale. Based on differences in the length of the CTPS2-derived amplicons, F. asiaticum, F. meridionale, and other members of the $F$. graminearum clade can now be distinguished (Fig. 2).

In the partial sequence of the TEF- $1 \alpha$ gene, there is cytosine at nt 364 in $F$. graminearum sensu stricto isolates while other species have a thymidine at this position. In addition, $F$. acaciaemearnsii isolates have a thymidine in the partial sequence of the TEF-1 $\alpha$ gene at position 416, while all other species of the $F$. graminearum clade have a cytosine at this position.

Based on the SNPs in these two genes between different species within the $F$. graminearum clade, species-specific primers were designed. The primers designed on the ammonia ligase 2 gene are specific for $F$. asiaticum or F. meridionale, whereas those based on TEF- $1 \alpha$ are specific for $F$. graminearum sensu stricto or $F$. acaciae-mearnsii. An overview of the primers is given in Table 1.

Species determination by PCR. The competition PCR was performed on all isolates with a combination of primers FgCTPSf024, FgCTPSr536, Fg6CTPSf177, and FgCTPSr306 to detect $F$. asiaticum and $F$. meridionale and other members of the $F$. graminearum clade. In the PCR amplification, $10 \mathrm{ng}$ of fungal DNA was added to a total reaction volume of $20 \mu \mathrm{l}$ containing $2 \mu \mathrm{l}$ of $10 \times$ PCR-buffer, $200 \mu \mathrm{M}$ dNTPs, $0.4 \mathrm{U}$ of Taq-DNA polymerase (Roche, Mannheim, Germany), with the following primer concentrations; $0.4 \mu \mathrm{M}$ Fg6CTPSf177, $0.4 \mu \mathrm{M}$ FgCTPSr306, $0.2 \mu \mathrm{M}$ FgCTPSf024, and $0.2 \mu \mathrm{M}$ FCTPSr536. The PCR conditions used were: $94^{\circ} \mathrm{C}$ for $2 \mathrm{~min}$, followed by 35 cycles of $94^{\circ} \mathrm{C}$ for $1 \mathrm{~min}, 55^{\circ} \mathrm{C}$ for $30 \mathrm{~s}, 72^{\circ} \mathrm{C}$ for $50 \mathrm{~s}$, and a final extension at $72^{\circ} \mathrm{C}$ for $10 \mathrm{~min}$. PCR products were sized on $1 \%$ agarose gels. For isolates that did belong to the $F$. graminearum clade but not to $F$. asiaticum, a second competition PCR was performed using specific primers for $F$. meridionale based on the ammonia ligase 2 gene. In this PCR amplification, $10 \mathrm{ng}$ of fungal DNA were added to a total reaction volume of $20 \mu \mathrm{l}$ containing $2 \mu \mathrm{l}$ $10 \times$ PCR-buffer, $200 \mu \mathrm{M}$ dNTPs, $0.4 \mathrm{U}$ of Taq-DNA polymerase (Roche, Mannheim, Germany), with the following primer concentrations; $0.4 \mu \mathrm{M}$ Fg6CTPSf177, $0.4 \mu \mathrm{M}$ Fg2CTPSr306, $0.2 \mu \mathrm{M}$ FgCTPSf024, and $0.2 \mu \mathrm{M}$ FCTPSr536 with the PCR conditions described above. In addition, a competition PCR was conducted with a combination of primers based on the TEF- $1 \alpha$ gene. The PCR conditions were identical as above except for the primer concentrations: $0.1 \mu \mathrm{M}$ Fg7TEFf364, $0.1 \mu \mathrm{M}$ Fg5TEFr411, 0.2 $\mu \mathrm{M}$ FgTEFf124, and $0.2 \mu \mathrm{M}$ FgTEFr590. On isolates that did not belong to the $F$. graminearum clade, single primer pair PCRs were performed with the primers specific for other species as listed in Table 1 . In these PCRs, $0.2 \mu \mathrm{M}$ each primer pair was added to $20 \mu \mathrm{l}$ of volume reaction system. PCR reagent concentrations were similar as used in the duplex PCR. Thermal cycler conditions used were $94^{\circ} \mathrm{C}$ for $2 \mathrm{~min}$, followed by 35 cycles of $94^{\circ} \mathrm{C}$ for $1 \mathrm{~min}, 60^{\circ} \mathrm{C}$ for $30 \mathrm{~s}, 72^{\circ} \mathrm{C}$ for $1 \mathrm{~min}$, and a final extension at $72^{\circ} \mathrm{C}$ for $10 \mathrm{~min}$. Amplicons were sized on $1 \%$ agarose gels.

Chemotyping of Fusarium isolates. Thirty-three NRRL reference isolates with known mycotoxin profiles (21) were chemotyped by PCR using $0.2 \mu \mathrm{M}$ each of the ToxP1 and ToxP 2 primers (15) (Table 1). PCR reagents added to the reaction system were the same as above and the thermal cycler conditions were: $94^{\circ} \mathrm{C}$ for $2 \mathrm{~min}$, followed by 35 cycles of $94^{\circ} \mathrm{C}$ for $1 \mathrm{~min}, 55^{\circ} \mathrm{C}$ for $1 \mathrm{~min}, 72^{\circ} \mathrm{C}$ for $50 \mathrm{~s}$, and a final extension at $72^{\circ} \mathrm{C}$ for $10 \mathrm{~min}$. Subsequently, the chemotype of all Chinese isolates was determined using these primers.

In addition, the chemotype of a representative set of isolates was determined by a deoxynivalenol enzyme-linked immuno-

TABLE 1. The primer sequences and the sizes of the polymerase chain reaction (PCR) fragments used to identify the Fusarium isolates from the Barley Fusarium Bank collection

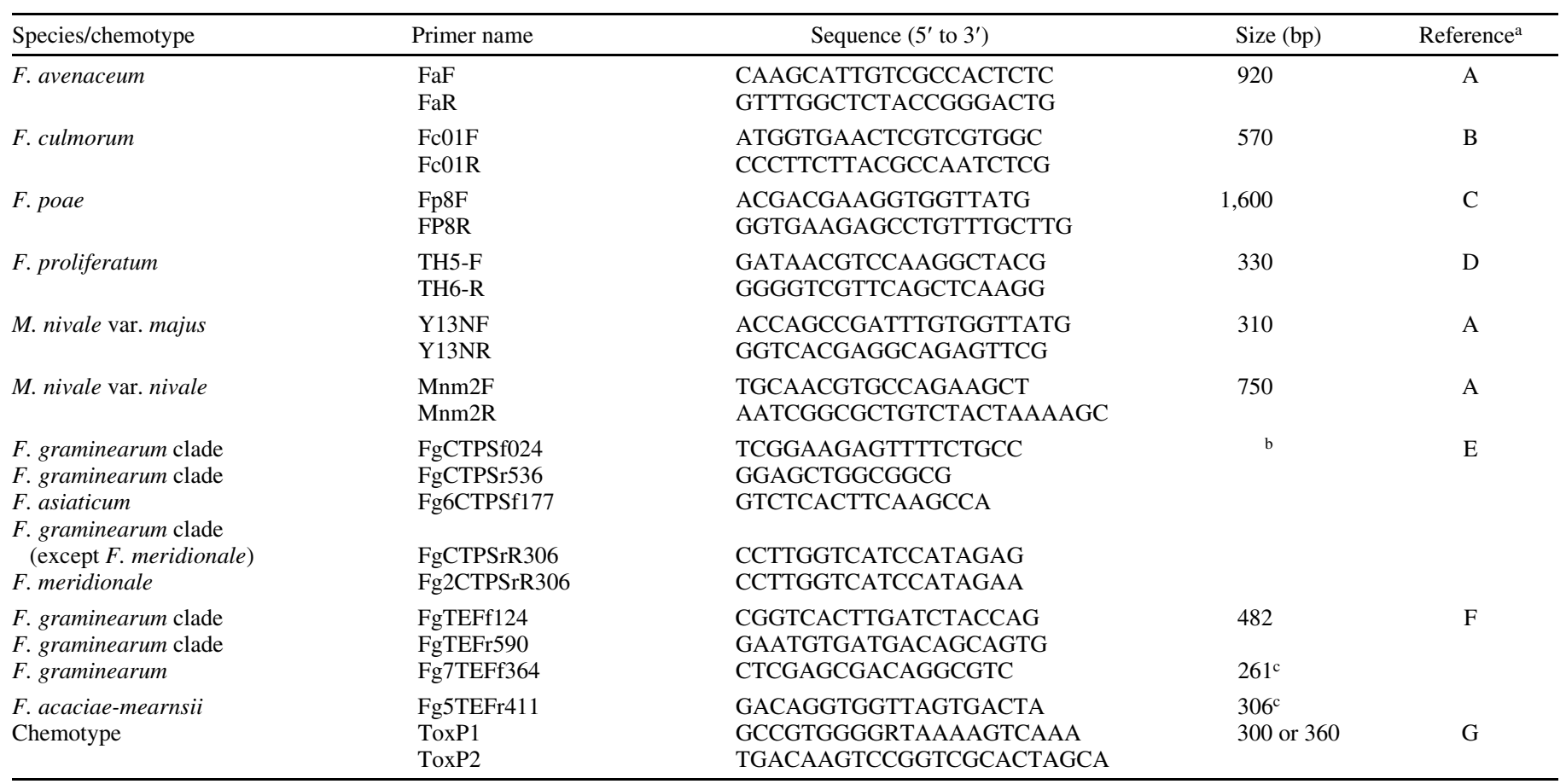

${ }^{a}$ References used: (A) Doohan et al. (8); (B) Nicholson et al. (20); (C) Parry and Nicholson (25); (D) Waalwijk et al. (30); (E) newly designed primers based on SNPs of ammonia ligase 2 gene (this study); (F) newly designed primers based on SNPs of TEF-1 $\alpha$ gene, and (G) Li et al. (15) where deoxynivalenol (DON) producers generate 300 -bp fragments and nivalenol (NIV) producers 360-bp fragments.

b Depending on the combination of primers, fragments of varying sizes can be expected: Fg6CTPSf177 and FgCTPSrR306 generate a 162-bp amplicon with $F$. asiaticum only; primers FgCTPSf024 and FgCTPSrR306 generate 311-bp fragments in all F. graminearum clade species except $F$. meridionale. Finally, FgCTPSf024 and FgCTPSr536 will lead to 536-bp fragments in all F. graminearum clade species. However, this fragment is not always visible due to competition with the 311- and 162-bp fragments.

${ }^{c}$ In combination with FgTEFf124, these primers generate fragments of 261 or 306 bp, respectively. 
sorbent assay (ELISA) (no. 5121DON[5]10.05 from Euro-Diagnostica, The Netherlands) according to the instructions of the manufacturer after growth on double autoclaved rice for 20 days at $25^{\circ} \mathrm{C}$ as described by Logrieco et al. (16). Although NIV has limited solubility in the extraction buffer used, the cross-reactivity of the antibodies in this kit towards NIV required a cut-off value for NIV producers of $700 \mu \mathrm{g} / \mathrm{g}$ (5\% of the maximum level of DON identified among the samples).

\section{RESULTS}

Collection of Fusarium isolates. A total of 1,894 isolates was collected by sampling fields in 23 counties in seven provinces and two municipalities (Table 2). Except for three counties in Jiangsu Province and the Huanggang County in Hubei Province, 20 or more isolates were collected. These isolates were stored in the largest collection of Fusarium isolates sampled from barley worldwide, collectively called the Barley Fusarium Bank (BFB). Information on the weather conditions of the last 4 weeks prior to sampling date, was obtained for one representative location per province. In general, humidity is high, around $65 \%$, in most provinces with the exception for Kunming, Yunnan, where the average humidity at noon is around $46 \%$. Precipitation can be more variable and as it was not recorded directly at the sampling fields, results of the weather station may not be representative.

All isolates were stored at $-80^{\circ} \mathrm{C}$. Twenty randomly selected isolates were checked for viability after 1 month storage, and all isolates tested grew after transfer from $-80^{\circ} \mathrm{C}$ to PDA plates.

Specificity of diagnostic Fusarium species PCR primers. NRRL isolates representing ten species used as a reference set in this study, showed the expected fragment sizes for the ammonia ligase 2 gene (Fig. 2). The primers for the ammonia ligase 2 gene produced fragments of $162,311,388$, and $536 \mathrm{bp}$ in isolates of F. asiaticum. The 311- and 536-bp fragments were observed in all other members of the $F$. graminearum clade except in $F$. meridionale. In F. meridionale, only the 536-bp fragment generated by the generic primers was observed. The only exception is isolate 28721 (F. asiaticum) which shows only the 536-bp fragment (Fig. 2B). This result is in agreement with previous reports because this isolate was shown to be a recombinant between $F$. asiaticum and F. meridionale (e.g., NRRL 28721 contains the F. meridionale
SNPs for the ammonia ligase 2 gene in a $F$. asiaticum background [21]). The 388- and 536-bp fragments are less intense in F. asiaticum and the 536-bp fragment is less intense in F. graminearum sensu stricto isolates, due to competition by the smaller fragments during the PCR (Fig. 2B).

With the primer mix based on the TEF- $1 \alpha$ gene, a 482-bp fragment was found in species belonging to the $F$. graminearum clade and fragments of $261 \mathrm{bp}$ in $F$. graminearum sensu stricto or $306 \mathrm{bp}$ in F. acaciae-mearnsii isolates. These results showed that the designed diagnostic primers were species-specific and can be used to study the Fusarium species diversity in the FHB complex in Chinese barley crops.

Species associated with FHB on barley. The 1,894 isolates of the BFB were characterized on the species level by the diagnostic PCR described previously. In this study, isolates belonging to the $F$. graminearum clade were detected and within this clade three species, F. asiaticum, F. graminearum sensu stricto, and F. meridionale were identified, as well as a small set of $F$. proliferatum isolates (Table 2). F. asiaticum was the dominant species and was found in all counties with a total of 1,706 isolates $(90.1 \%)$. We identified $45 \mathrm{~F}$. graminearum sensu stricto isolates $(2.4 \%)$, but only in the Hubei and Sichuan Provinces. F. meridionale was detected 27 times (1.4\%) in the upper reaches of Yangtze River (Sichuan, Chongqing, and Hubei; counties 3, 4, 7, and 9; Fig. 1). In total, 68 isolates $(3.6 \%)$ appeared to belong to the F. graminearum clade, but based on two diagnostic primer sets we could not determine to which species since no specific fragments for either $F$. asiaticum, F. graminearum sensu stricto, F. acaciaemearnsii, or $F$. meridionale were obtained. These isolates were sampled in all provinces and municipalities (Table 2). F. proliferatum was isolated seven times from a single county (Wuchang) in the Hubei province. In each province or municipality sampled, some isolates were found that could not be identified. In total 41 isolates $(2.2 \%)$ did not show any fragment with any of the Fusarium primers used.

The results showed that the species complexity of FHB in the lower valleys of the Yangtze River is limited; in fact all the 607 isolates collected east of Hubei province (counties 13 to 23, Fig. 1) that could be determined at the species level, were $F$. asiaticum. In the upper valleys (counties 1 to 12 , Fig. 1), F. asiaticum was again the dominant species, but in Sichuan, Chongqing, and

TABLE 2. Sampling sites of Fusarium isolates along the Yangtze River including GPS data, altitude, and the distribution of Fusarium spp.

\begin{tabular}{|c|c|c|c|c|c|c|c|c|c|c|c|c|}
\hline $\begin{array}{l}\text { Province/ } \\
\text { municipality }\end{array}$ & $\begin{array}{l}\text { Site } \\
\text { no. }\end{array}$ & County & Latitude & Longitude & $\begin{array}{l}\text { Altitude } \\
\text { (m) }\end{array}$ & $\begin{array}{c}F . \\
\text { asiaticum }\end{array}$ & $\begin{array}{c}F \\
\text { graminearum }\end{array}$ & $\begin{array}{c}F \\
\text { meridionale }\end{array}$ & $\begin{array}{c}\text { F. graminearum } \\
\text { clade } \\
\text { unspecified }\end{array}$ & $\begin{array}{c}F . \\
\text { proliferatum }\end{array}$ & ND & Total \\
\hline \multirow[t]{2}{*}{ Yunnan } & 1 & Baoshan & $25^{\circ} 07^{\prime}$ & $99^{\circ} 09^{\prime}$ & 1,693 & 20 & 0 & 0 & 0 & 0 & 0 & 20 \\
\hline & 2 & Yuxi & $24^{\circ} 21^{\prime}$ & $102^{\circ} 32^{\prime}$ & 1,639 & 24 & 0 & 0 & 0 & 0 & 5 & 29 \\
\hline \multirow[t]{3}{*}{ Sichuan } & 3 & Mianzhu & $31^{\circ} 19^{\prime}$ & $104^{\circ} 12^{\prime}$ & 593 & 108 & 7 & 1 & 0 & 0 & 4 & 120 \\
\hline & 4 & Mianyang & $31^{\circ} 28^{\prime}$ & $104^{\circ} 46^{\prime}$ & 453 & 71 & 0 & 3 & 5 & 0 & 0 & 79 \\
\hline & 5 & Xingsheng & $30^{\circ} 55^{\prime}$ & $105^{\circ} 02^{\prime}$ & 451 & 114 & 21 & 0 & 11 & 0 & 2 & 148 \\
\hline \multirow[t]{2}{*}{ Chongqing } & 6 & Tongnan & $30^{\circ} 13^{\prime}$ & $105^{\circ} 47^{\prime}$ & 263 & 34 & 0 & 0 & 0 & 0 & 0 & 34 \\
\hline & 7 & Yongchuan & $29^{\circ} 21^{\prime}$ & $105^{\circ} 53^{\prime}$ & 301 & 136 & 0 & 8 & 0 & 0 & 2 & 146 \\
\hline \multirow[t]{5}{*}{ Hubei } & 8 & Maliang & $30^{\circ} 52^{\prime}$ & $112^{\circ} 31^{\prime}$ & 52 & 113 & 17 & 0 & 1 & 0 & 3 & 134 \\
\hline & 9 & Shayang & $30^{\circ} 41^{\prime}$ & $112^{\circ} 28^{\prime}$ & 56 & 146 & 0 & 15 & 2 & 0 & 0 & 163 \\
\hline & 10 & Jingzhou & $30^{\circ} 35^{\prime}$ & $112^{\circ} 19^{\prime}$ & 36 & 100 & 0 & 0 & 20 & 0 & 2 & 122 \\
\hline & 11 & Wuchang & $30^{\circ} 19^{\prime}$ & $114^{\circ} 07^{\prime}$ & 20 & 219 & 0 & 0 & 1 & 7 & 5 & 232 \\
\hline & 12 & Huanggang & $30^{\circ} 26^{\prime}$ & $114^{\circ} 47^{\prime}$ & 24 & 14 & 0 & 0 & 0 & 0 & 0 & 14 \\
\hline Anhui & 13 & Huaiyuan & $32^{\circ} 57^{\prime}$ & $117^{\circ} 11^{\prime}$ & 9 & 36 & 0 & 0 & 2 & 0 & 1 & 39 \\
\hline \multirow[t]{4}{*}{ Jiangsu } & 14 & Nanjing & $32^{\circ} 03^{\prime}$ & $118^{\circ} 46^{\prime}$ & 2 & 8 & 0 & 0 & 2 & 0 & 0 & 10 \\
\hline & 15 & Nantong & $32^{\circ} 05^{\prime}$ & $121^{\circ} 03^{\prime}$ & 2 & 1 & 0 & 0 & 0 & 0 & 0 & 1 \\
\hline & 16 & Yancheng & $31^{\circ} 25^{\prime}$ & $120^{\circ} 46^{\prime}$ & 15 & 38 & 0 & 0 & 2 & 0 & 3 & 43 \\
\hline & 17 & Wujiang & $31^{\circ} 18^{\prime}$ & $120^{\circ} 37^{\prime}$ & 3 & 5 & 0 & 0 & 1 & 0 & 1 & 7 \\
\hline \multirow[t]{2}{*}{ Zhejiang } & 18 & Jiaxing & $30^{\circ} 45^{\prime}$ & $120^{\circ} 44^{\prime}$ & 6 & 107 & 0 & 0 & 10 & 0 & 3 & 120 \\
\hline & 19 & Hangzhou & $30^{\circ} 15^{\prime}$ & $120^{\circ} 10^{\prime}$ & 17 & 258 & 0 & 0 & 1 & 0 & 3 & 262 \\
\hline \multirow[t]{2}{*}{ Shanghai } & 20 & Yongfeng & $30^{\circ} 55^{\prime}$ & $121^{\circ} 38^{\prime}$ & 8 & 85 & 0 & 0 & 10 & 0 & 1 & 96 \\
\hline & 21 & Nongkeyuan & $31^{\circ} 06^{\prime}$ & $121^{\circ} 68^{\prime}$ & 4 & 7 & 0 & 0 & 0 & 0 & 0 & 7 \\
\hline \multirow[t]{2}{*}{ Fujian } & 22 & Fuzhou & $26^{\circ} 03^{\prime}$ & $119^{\circ} 18^{\prime}$ & 5 & 37 & 0 & 0 & 0 & 0 & 6 & 43 \\
\hline & 23 & Putian & $25^{\circ} 26^{\prime}$ & $119^{\circ} 00^{\prime}$ & 9 & 25 & 0 & 0 & 0 & 0 & 0 & 25 \\
\hline Total & & & & & & 1,706 & 45 & 27 & 68 & 7 & 41 & 1,894 \\
\hline
\end{tabular}


Hubei, $F$. graminearum sensu stricto and/or $F$. meridionale were also found. The occurrence of $F$. graminearum sensu stricto and F. meridionale along the Yangtze River shows statistically significant differences for the different regions. The probability of the number of $F$. graminearum sensu stricto isolates found assuming an equal distribution along the Yangtze River as determined by the chi-square test is low for the provinces Sichuan and Zhejiang with $P<0.0001$ and 0.0025 , respectively. Similarly, the probability of the number of $F$. meridionale isolates found assuming an equal distribution along the Yangtze River, as determined by the chisquare test, is low for the provinces Sichuan $(P<0.0001)$ and Zhejiang $(P<0.001)$, respectively. Members of the $F$. graminearum clade that could not further be identified were collected in all sampled regions, including the lower valleys and made up $3.6 \%$ (68 of the 1,894$)$ of the total number of isolates.

Chemotype diversity. To determine whether ToxP1/ToxP2 primers could discriminate between DON and NIV chemotypes among type B trichothecenes producing Fusarium species, we performed a PCR with primers ToxP1/ToxP2 on 33 NRRL isolates. The results were consistent with their documented chemotypes based on chemical analyses (32). A selected set of Chinese isolates that showed either DON or NIV chemotypes by PCR were further tested by ELISA. Among 49 isolates, 48 showed the same chemotype in the PCR and ELISA analyses. The remaining Chinese isolate, bfb0792, showed a high ELISA value, while the PCR was indicative for an NIV chemotype. This isolate was also analyzed with diagnostic primers designed on the tri3 and tri12 genes (13,32), which confirmed the NIV chemotype obtained with the ToxP primers. This indicated that the ToxP1/ToxP2 primers were reliable and consistent with other primers currently in use for chemotyping. Subsequently, the chemotypes of the $\mathrm{Fu}$ sarium population sampled from barley in China were determined using the ToxP1/ToxP2 primers. The results are summarized in Table 3 . Of the $1,846 \mathrm{~F}$. graminearum clade isolates, 1,192 isolates yielded a DON-specific PCR fragment and 642 isolates had an NIV-specific fragment, which indicated that DON is the predominant mycotoxin produced in the barley crops in the sampled regions. Although the DON chemotype is dominant in F. asiaticum, NIV had a relatively higher proportion in $F$. asiaticum than in other species. Of the 1,706 F. asiaticum isolates, 1,064 isolates were of the DON chemotype $(62.4 \%)$ and 630 were NIV producers. In F. meridionale, $81.5 \%$ of the isolates showed the DON chemotype, and 44 of $45 \mathrm{~F}$. graminearum sensu stricto isolates $(97.7 \%)$ were of the DON chemotype. Among the 68 unspecified F. graminearum clade isolates, 62 isolates produced a DON chemotype (91.2\%) specific PCR fragment, and six were categorized to the NIV chemotype.
The geographical distribution of chemotypes showed dramatic differences (Table 3 ). Within the $F$. asiaticum populations, the NIV chemotype was remarkably more frequent in southwest China (counties 1 to 7, Fig. 1) compared with the lower reaches of the Yangtze River (counties 8 to 23, Fig. 1). None of the 44 isolates collected in Yunnan province were categorized to the DON chemotype, while in Sichuan and Chongqing, the DON chemotype accounted for 5 and $19 \%$, respectively (Fig. 3). In contrast to these mountainous sampling sites ( $>250 \mathrm{~m}$ above sea level), in all other provinces the DON chemotype accounted for more than $70 \%$. In nearly all provinces, the number of DON and NIV producers found deviated significantly from the expected number, based on the assumption of equal frequencies of DON and NIV producers in all provinces as tested by a chi-square test (Table 3 ). In addition, $F$. graminearum sensu stricto isolates collected in Sichuan province did not show the DON NIV ratio found for $F$. asiaticum collected from the same province (Table 3 ). Only 1 out of $28 \mathrm{~F}$. graminearum sensu stricto isolates from Sichuan province showed the NIV chemotype, which is dominant in $F$. asiaticum isolates from Sichuan province (chi-square test; $P<0.0001$, Table 3 ).

\section{DISCUSSION}

FHB is a devastating disease of wheat, barley, and other small grain cereals all over the world. Particularly in China, FHB is a major constraint to wheat production (17). To take the appropriate agronomic measures, population dynamic studies on the pathogen are essential. This study is the first comprehensive survey on barley in China in an attempt to resolve the complex and important problem of FHB in barley and cereals in general. Population studies are particularly important in perspective of the suggested increased disease pressure of Fusarium that became apparent by the shift of farmers from wheat to other crops due to increased FHB. In Hubei, the high incidence of FHB on wheat and the increasing demand for barley for food and feed prompted farmers to switch from wheat to other crops, such as barley. However, although the visual symptoms may not always be clear and the yield losses are lower, barley is also susceptible to Fusarium and consequently contamination with mycotoxins is a major threat in barley as well. Since FHB is caused by a complex of species, we first addressed which Fusarium species occur in barley. Here we present the isolation and characterization of a large collection of Fusarium isolates from barley kernels.

In the last decades, a number of surveys were made in China on the occurrence of fungal species associated with FHB on wheat. In these large surveys, isolates were not stored and Fusarium species were diagnosed only morphologically. In those studies $F$.

TABLE 3. Distribution of deoxynivalenol (DON) and nivalenol (NIV) chemotypes among Fusarium species from nine provinces along the Yangtze River as determined by the ToxP-PCR

\begin{tabular}{|c|c|c|c|c|c|c|c|c|c|c|}
\hline \multirow[b]{2}{*}{ Province/municipality } & \multicolumn{4}{|c|}{ F. asiaticum } & \multicolumn{2}{|c|}{ F. meridionale } & \multicolumn{2}{|c|}{ F. graminearum s.s. } & \multicolumn{2}{|c|}{$\begin{array}{l}\text { F. graminearum clade } \\
\text { unspecified }^{\mathrm{a}}\end{array}$} \\
\hline & DON & NIV & $\mathrm{ND}^{\mathrm{b}}$ & Sig. ${ }^{c}$ & DON & NIV & DON & NIV & DON & NIV \\
\hline Yunnan & 0 & 44 & & $* * *$ & & & & & & \\
\hline Chongqing & 29 & 139 & 2 & $* * *$ & 8 & & & & & \\
\hline Hubei & 538 & 51 & 3 & $* * *$ & 14 & 1 & 17 & & 24 & \\
\hline Anhui & 26 & 10 & & NS & & & & & 2 & \\
\hline Fujian & 54 & 5 & 3 & $* * *$ & & & & & & \\
\hline Total & 1,064 & 630 & 12 & & 22 & 5 & 44 & 1 & 62 & 6 \\
\hline
\end{tabular}

a $F$. graminearum clade unspecified indicates that the isolates are members the F. graminearum clade sensu O'Donnell $(21,22)$.

${ }^{\mathrm{b}} \mathrm{ND}$, not determined as no fragment was obtained.

${ }^{\mathrm{c}}$ Likelihood, determined by the chi-square test, that the observed numbers of DON and NIV producers occur, assuming that the ratio of DON and NIV producers is equal in all provinces: $* * * P<0.0001$.

${ }^{\mathrm{d}}$ Deviates significantly for the ratio found for F. asiaticum $(P<0.0001)$. 
graminearum was found to be the predominant species occurring on wheat in China (17). However, the new phylogenetic analyses demonstrated the presence of nine distinct species in the microscopically monomorphic $F$. graminearum clade (21). We developed species specific primers for the most important species of the F. graminearum clade reported to be present in China (22). Two newly developed diagnostic PCRs based on competition PCR could discriminate between several species ( $F$. asiaticum, $F$. meridionale, F. graminearum sensu stricto, and F. acaciae-mearnsii) within the $F$. graminearum clade. In the present study, these duplex primer sets and other documented primers were used to differentiate isolates sampled from barley along the Yangtze River just before harvest in 2005. Results showed that $F$. asiaticum (lineage 6 of the $F$. graminearum clade as defined by O'Donnell et al., [20]) is predominant, consistent with results from previous surveys in terms of the $F$. graminearum clade $(9,15)$. Other species, such as $F$. meridionale and $F$. graminearum sensu stricto, at lower proportions were also associated with FHB in barley. Regional differences are large even between sampling locations within the same province and statistically significant. The populations in the lower valleys (Anhui, Jiangsu, Shanghai, and Zhejiang) and in Fujian Province are less diverse than populations in the upper and middle regions of Yangtze River (Yunnan, Sichuan, Chongqing, and Hubei). In the upper and middle regions, $45 \mathrm{~F}$. graminearum sensu stricto isolates, $27 \mathrm{~F}$. meridionale isolates, and seven $F$. proliferatum isolates were identified while in the lower valley and in Fujian Province all these species were absent. These results are consistent with the results of Gale et al. (9), who showed that all 225 isolates from four different wheat fields in Zhejiang province belonged to F. asiaticum. Similar results were obtained in Wuhan, where all 192 isolates obtained from a single wheat field were $F$. asiaticum (29). Surprisingly, $F$. avenaceum, $F$. culmorum, and $F$. poae that are more often associated with FHB in cooler, wetter regions (25), were not detected among the 1,894 isolates recovered from barley.

By means of PCR with primers ToxP1/ToxP2, we detected DON- and NIV-producing isolates in every sampling place except for Yunnan province, where only the NIV chemotype was found. The distribution of type B trichothecenes producers in the present study revealed highly significant differences between provinces. $F$. asiaticum isolates with the DON chemotype dominate the lower valleys of Yangtze River, which is in agreement with previously published work $(11,15)$, whereas NIV producers are dominant in the upper valleys. This was also found by $\mathrm{Li}$ et al. (15), but these authors collected only 3 (out of 3) NIV producing isolates in these areas. In contrast, nearly all the $F$. graminearum isolates collected produced DON (44 out of 45). The situation in Sichuan Province was particularly remarkable, since NIV-producing $F$. asiaticum and DON-producing F. graminearum were collected on the same day from the same crop in a single field. This would indicate that, although forced laboratory crosses have been reported (12), these species are sympatric.

No clear correlation with species diversity or ratios between DON and NIV chemotypes was found with the cropping system
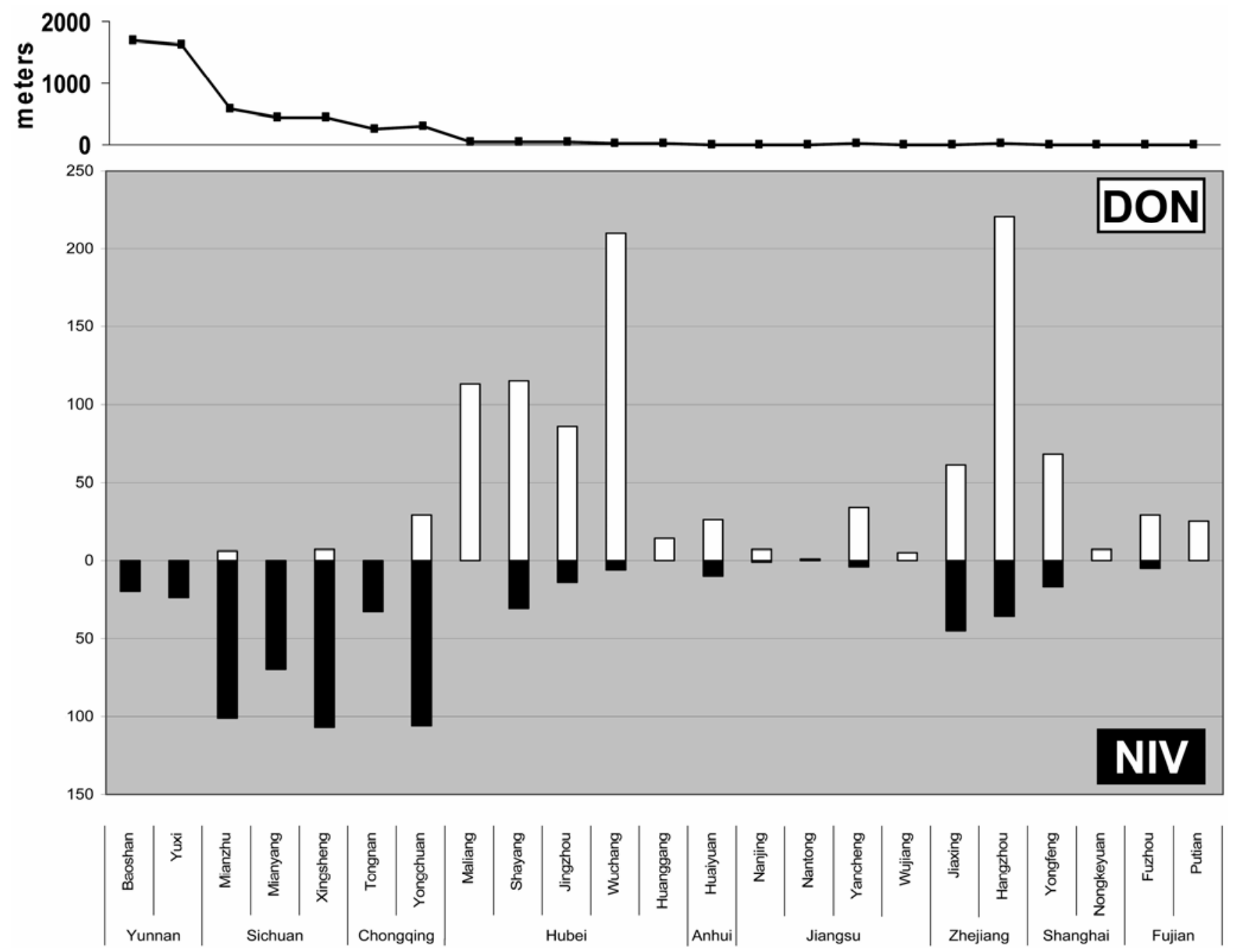

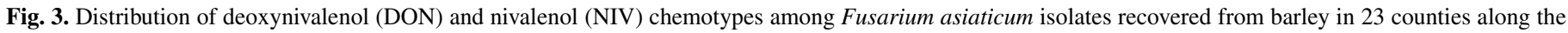

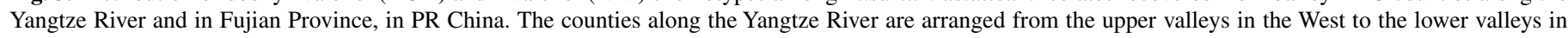
the East. 
or climatic conditions. In the lower valleys, the crop rotation is simple (mainly rice), whereas it is more diverse in the southwest part of China, where besides rice, wheat and corn are grown on a large scale. Minimum and maximum temperatures as well as humidity show large variations during the 4 weeks preceding the collection of spikes. However, for temperature, humidity or rain fall, no clear grouping of the provinces Yunnan, Sichuan, and Chongqing versus Hubei, Anhui, Jiangsu, Shanghai, Zhejiang, and Fujian could be found. Most variation in the climatic conditions could be found in Yunnan as opposed to the other provinces, since in Yunnan lower temperatures and lower humidities were recorded. In Fujian, on the other hand, higher temperatures and higher humidities were recorded prior to sampling, but the differences with other provinces are limited compared with the difference between Yunnan and the other provinces.

The current distribution of chemotypes and species is reminiscent of a recent migration. The altitude of the sampling site showed a very strong correlation with the type of mycotoxin produced (Fig. 3). Yunnan, with elevations $>1500 \mathrm{~m}$ and Sichuan and Chongqing, surrounded by large mountain ridges, may reflect isolated regions. It is likely that these mountains represent geographical barriers for the migration of isolates and the original population is retained longer in these isolated regions, as was found for other pathogens such as Plasmopara viticola (10). We hypothesize that the isolates of the mountain rich provinces represent the old population and that NIV-producing $F$. asiaticum may have dominated Chinese FHB populations in the past and have been replaced by DON producers. In addition, the Fusarium population causing FHB on wheat and barley may have contained more $F$. graminearum which could be overtaken by $F$. asiaticum. What could be the driving force of this displacement? Possibly $F$. asiaticum-producing DON is more aggressive on wheat. Results of Maier et al. (18) indicate that compared to NIV, DON is a stronger virulence factor on wheat. Therefore such a displacement may have caused the increased problems of FHB on wheat observed in the last decade. Although this is currently only a hypothesis, we recommend monitoring populations elsewhere for shifts among the species causing FHB and shifts towards $F$. asiaticum in particular. Currently we are testing the virulence of $F$. asiaticum and $F$. graminearum isolates producing either DON or NIV on both wheat and barley to determine whether we can identify differences in virulence among species and within species among chemotypes.

Our results represent the first analysis of a comprehensive collection of Fusarium isolates from barley in China. To our knowledge this BFB collection of Fusarium isolates obtained from barley is the largest in the world. The size and the hierarchical sampling in one season allowed detailed analyses of the species causing FHB along the Yangtze River. Similar to previous studies performed on wheat, we identified $F$. asiaticum as the predominant species. Unlike previous reports our data suggest that the Fusarium population along the Yangtze River is not a single metapopulation, but that highly significant regional differences occur. This is particularly obvious for the chemotype. Why this dramatic gradient occurs is not yet clear. It may be due to differences in climatic conditions or to the crops used in the rotation, or we may have uncovered a recent migration. Further characterization of the isolates and comparison with isolates from different years and crops are required to get a better understanding of the cause and stability of these substructures in Fusarium populations.

\section{ACKNOWLEDGMENTS}

This work was financed by KNAW-MOST (04-PSA-BD-05) and by the State Basic Theory Research of China (973 program, grant No. 2004CB720407). We thank M. Li from the Food Crop Institute, Hubei Academy of Agricultural Science for valuable contributions to sampling of Fusarium isolates, K. O'Donnell for the NRRL isolates used as a reference set of Fusarium isolates and, I. de Vries for technical contributions.

\section{LITERATURE CITED}

1. Bai, G. H., and Shaner, G. 1996. Variation in Fusarium graminearum and cultivar resistance to wheat scab. Plant Dis. 80:975-980.

2. Bottalico, A., and Perrone, G. 2002. Toxigenic Fusarium species and mycotoxins associated with head blight in small-grain cereals in Europe. Eur. J. Plant Pathol. 108:611-624.

3. Carter, J. P., Rezanoor, H. N., Desjardins, A. E., and Nicholson, P. 2000.Variation in Fusarium graminearum isolates from Nepal associated with their host of origin. Plant Pathol. 49:452-460.

4. Carter, J. P., Rezanoor, H. N., Holden, D., Desjardins, A. E., Plattner, R. D., and Nicholson, P. 2002.Variation in pathogenicity associated with the genetic diversity of Fusarium graminearum. Eur. J. Plant Pathol. 108:573583.

5. Chandler, E. A., Duncan, R. S., Thomsett, M. A., and Nicholson, P. 2003. Development of PCR assays to tri7 and tri13 and characterisation of chemotypes of Fusarium graminearum, Fusarium culmorum and Fusarium cerealis. Physiol. Mol. Plant Pathol. 62:355-367.

6. CWSCG. 1984. Fusarium species distribution and pathogenicity from scabby heads in China. J. Shanghai Normal Coll. 3:69-82.

7. D’Mello, J. P. F., Placinta, C. M., and Macdonald, A. M. C. 1999. Fusarium mycotoxins. A review of global implications for animal health, welfare and productivity. Anim. Feed Sci.Technol. 80:183-205.

8. Doohan, F. M., Parry, D. W., Jenkinson, P., and Nicholson, P. 1998. The use of species-specific PCR-based assays to analyse Fusarium ear blight of wheat. Plant Pathol. 47:197-205

9. Gale, L. R., Chen, L. F., Hernick, C. A., Takamura, K., and Kistler, H. C. 2002. Population analysis of Fusarium graminearum from wheat fields in eastern China. Phytopathology 92:1315-1322.

10. Gobbin, D., Rumbou, A., Linde, C. C., and Gessler, C. 2006. Population genetic structure of Plasmopara viticola after 125 years of colonization in European vineyards. Mol. Plant Pathol. 7:519-531.

11. Ji, L., Cao, K., Hu, T., and Wang, S. 2007. Determination of deoxynivalenol and nivalenol chemotypes of Fusarium graminearum isolates from China by PCR assay. J. Phytopathol. 155:505-512.

12. Jurgenson, J. E., Bowden, R. L., Zeller, K. A., Leslie, J. F., Alexander, N. J., and Plattner, R. D. 2002. A Genetic Map of Gibberella zeae (Fusarium graminearum). Genetics 160:1451-1460.

13. Jennings, P., Coates, M. E., Turner, J. A., Chandler, E. A., and Nicholson, P. 2004. Determination of deoxynivalenol and nivalenol chemotypes of Fusarium culmorum isolates from England and Wales by PCR assay. Plant Pathol. 53:182-190.

14. Lee, T., Oh, D. W., Kim, H. S., Lee, J., Kim, Y. H., Yun, S. H., and Lee, Y. W. 2001. Identification of deoxynivalenol- and nivalenol-producing chemotypes of Gibberella zeae by using PCR. Appl. Environ. Microbiol. 67:2966-2972.

15. Li, H. P., Wu, A. B., Zhao, C. S., Scholten, O., Löffler, H., and Liao, Y. C. 2005. Development of a generic PCR detection of deoxynivalenol- and nivalenol-chemotypes of Fusarium graminearum. FEMS Microbiol. Lett. 243:505-511.

16. Logrieco, A., Rizzo, A., Ferracane, R., and Ritieni, A. 2002. Occurrence of beauvericin and enniatins in wheat affected by Fusarium avenaceum head blight. Appl. Environ. Microbiol. 68:82-85.

17. Lu, W. H., Chen, S. H., and Wang, Y. Z. 2001. Research on Fusarium Head Scab. Science Publisher, Beijing.

18. Maier, F. J., Miedaner, T., Hadeler, B., Felk, A., Salomon, S., Lemmens, M., Kassner, H., and Schaefer, W. 2006. Involvement of trichothecenes in fusarioses of wheat, barley and maize evaluated by gene disruption of the trichodiene synthase (tri5) gene in three field isolates of different chemotype and virulence. Mol. Plant Pathol. 7:449-461.

19. McMullen, M., Jones, R., and Gallenberg, D. 1997. Scab of wheat and barley: A re-emerging disease of devastating impact. Plant Dis. 81:13401348.

20. Nicholson, P., Simpson, D. R., Weston, G., Rezanoor, H. N., Lees, A. K., Parry, D. W., and Joyce, D. 1998. Detection and quantification of Fusarium culmorum and Fusarium graminearum in cereals using PCR assays. Physiol. Mol. Plant Pathol. 53:17-37.

21. O’Donnell, K., Kistler, H. C., Tacke, B. K., and Casper, H. H. 2000.Gene genealogies reveal global phylogeographic structure and reproductive isolation among lineages of Fusarium graminearum, the fungus causing wheat scab. P. Natl Acad. Sci. USA 97:7905-7910.

22. O’Donnell, K., Ward, T. J., Geiser, D. M., Kistler, H. C., and Aoki, T. 2004. Genealogical concordance between the mating type locus and seven other nuclear genes supports formal recognition of nine phylogenetically distinct species within the Fusarium graminearum clade. Fungal Genet. Biol. 41:600-623. 
23. Oerke, E. C. 2006. Spatial distribution of Fusarium spp. causing head blight in wheat fields. 9th European Fusarium Seminar, Wageningen, The Netherlands.

24. Parry, D. W., Jenkinson, P., and Mcleod, L. 1995. Fusarium ear blight (scab) in small grain cereals-a review. Plant Pathol. 44:207-238.

25. Parry, D. W., and Nicholson, P. 1996. Development of a PCR assay to detect Fusarium poae in wheat. Plant Pathol. 45:383-391.

26. Placinta, C. M., D'Mello, J. P. F., and Macdonald, A. M. C. 1999. A review of worldwide contamination of cereal grains and animal feed with Fusarium mycotoxins. Anim. Feed Sci. Technol.78:21-37.

27. Schlatter, J. 2004. Toxicity data relevant for hazard characterization. Toxicol. Lett. 153:83-89

28. Tekauz, A., McCallum, B., and Gilbert, J. 2000. Review: Fusarium head blight of barley in western Canada. Can. J. Plant Pathol. 22:9-16.

29. Waalwijk, C., de Vries, I., Köhl, J., Xu, X., van der Lee, T., and Kema, G. H. J. 2008. Development of quantitative detection methods for Fusarium in cereals and their application. Pages 197-207 in: Mycotoxins: Detection
Methods, Management, Public Health and Agricultural Trade. J. F. Leslie, R. Bandyopadhyay, and A. Visconti, eds. CABI Publishing.

30. Waalwijk, C., Kastelein, P., de Vries, I., Kerényi, Z., van der Lee, T., Hesselink, T., Köhl, J., and Kema, G. H. J. 2003. Major changes in Fusarium spp. in wheat in the Netherlands. Eur. J. Plant Pathol. 109:743-754.

31. Waalwijk, C., van der Heide, R., de Vries, P. M., van der Lee, T. A. J., Schoen, C. D., Costrel-de Corainville, G., Häuser-Hahn, I., Kastelein, P., Köhl, J., Lonnet, P., Demarquet, T., and Kema, G. H. J. 2004. Quantitative detection of Fusarium species in wheat using Taqman. Eur. J. Plant Pathol. 110:481-494.

32. Ward, T. J., Bielawski, J. P., Kistler, H. C., Sullivan, E., and O’Donnell, K. 2002. Ancestral polymorphism and adaptive evolution in the trichothecene mycotoxin gene cluster of phytopathogenic Fusarium. Proc. Natl. Acad. Sci. USA 99:9278-9283.

33. Windels, C. E. 2000. Economic and social impacts of Fusarium head blight: Changing farms and rural communities in the Northern Great Plains. Phytopathology 90:17-21. 